\title{
Effect of tendon vibration during wide- pulse neuromuscular electrical stimulation (NMES) on muscle force production in people with spinal cord injury $(\mathrm{SCl})$
}

Vanesa Bochkezanian ${ }^{1,2,3^{*}}$ (D, Robert U. Newton ${ }^{2,3,4}$, Gabriel S. Trajano ${ }^{5}$, Amilton Vieira ${ }^{6}$, Timothy S. Pulverenti ${ }^{3}$ and Anthony J. Blazevich ${ }^{3}$

\begin{abstract}
Background: Neuromuscular electrical stimulation (NMES) is commonly used in skeletal muscles in people with spinal cord injury (SCI) with the aim of increasing muscle recruitment and thus muscle force production. NMES has been conventionally used in clinical practice as functional electrical stimulation (FES), using low levels of evoked force that cannot optimally stimulate muscular strength and mass improvements, and thus trigger musculoskeletal changes in paralysed muscles. The use of high intensity intermittent NMES training using wide-pulse width and moderate-intensity as a strength training tool could be a promising method to increase muscle force production in people with $\mathrm{SCl}$. However, this type of protocol has not been clinically adopted because it may generate rapid muscle fatigue and thus prevent the performance of repeated high-intensity muscular contractions in paralysed muscles. Moreover, superimposing patellar tendon vibration onto the wide-pulse width NMES has been shown to elicit further increases in impulse or, at least, reduce the rate of fatigue in repeated contractions in able-bodied populations, but there is a lack of evidence to support this argument in people with SCl.

Methods: Nine people with $\mathrm{SCl}$ received two NMES protocols with and without superimposing patellar tendon vibration on different days (i.e. STIM and STIM+vib), which consisted of repeated $30 \mathrm{~Hz}$ trains of 58 wide-pulse width $(1000 \mu \mathrm{s})$ symmetric biphasic pulses (0.033-s inter-pulse interval; $2 \mathrm{~s}$ stimulation train; 2 -s inter-train interval) being delivered to the dominant quadriceps femoris. Starting torque was $20 \%$ of maximal doublet-twitch torque and stimulations continued until torque declined to $50 \%$ of the starting torque. Total knee extensor impulse was calculated as the primary outcome variable.
\end{abstract}

Results: Total knee extensor impulse increased in four subjects when patellar tendon vibration was imposed (59.2 $\pm 15.8 \%)$ but decreased in five subjects $(-31.3 \pm 25.7 \%)$. However, there were no statistically significant differences between these sub-groups or between conditions when the data were pooled.

Conclusions: Based on the present results there is insufficient evidence to conclude that patellar tendon vibration provides a clear benefit to muscle force production or delays muscle fatigue during wide-pulse width, moderateintensity NMES in people with SCl.

Trial registration: ACTRN12618000022268. Date: 11/01/2018. Retrospectively registered.

\footnotetext{
* Correspondence: v.bochkezanian@cqu.edu.au; vanesaboch@gmail.com

'Department of Exercise and Health Sciences, School of Health, Medical and

Applied Sciences, Central Queensland University, Building 34.1.02, Bruce

Highway, North Rockhampton, Qld 4702, Australia

${ }^{2}$ Exercise Medicine Research Clinic, Edith Cowan University, Perth, Australia

Full list of author information is available at the end of the article
} 


\section{Background}

Spinal cord injury (SCI) is most commonly caused by trauma and interrupts the connection between supraspinal and spinal regions of the CNS [1]. This leads to a reduction of the voluntary activation of muscles below the lesion level, reducing muscle force production, impairing physical function, and profoundly compromising physical health and quality of life (QoL) [1-3]. Furthermore, the reduction in muscle force production is a major predictor of mortality risk and seems to be partly attributable to the quantity (i.e. absolute muscle volume) and quality (i.e. muscle density) of muscle mass $[4,5]$.

A common method to increase muscular force production and muscle mass is the use of neuromuscular strength training. Particularly, high-intensity muscle contractions have been proven to enhance longevity and QoL in different clinical populations, such as diabetes type 2 and osteoporosis, as well as in older adults [6-9]. However, muscle strength training poses an increased challenge for people with a neurological condition, such as people with SCI. Alternatively, neuromuscular electrical stimulation (NMES) is a commonly used intervention in rehabilitation programs to increase muscle recruitment and thus muscle force production, especially in individuals with a complete loss of motor function [10-12]. NMES has been conventionally used in clinical practice as functional electrical stimulation (FES), i.e. a prolonged and low levels of evoked force NMES exercise paired simultaneously or intermittently with a functional task [12]. However, such interventions cannot optimally stimulate muscular strength and mass improvements, imposing a higher load to the muscle to obtain higher force output $[13,14]$ in accordance with the overload training principle [15], to obtain musculoskeletal changes in paralysed muscles.

The use of high intensity intermittent NMES training (hereafter referred to as near-maximal evoked muscle contractions) as a strength training tool has not been adopted clinically in people with SCI, probably due to the lack of evidence from long-term intervention trials documenting its physiological effects $[16,17]$ but mainly because these types of protocols generate rapid muscle fatigue and thus prevent the performance of repeated high-intensity muscular contractions [18-21]. This muscle fatigue is exacerbated in people with SCI due to the loss of fatigue-resistant motor units and their higher proportion of more fatigable motor units [22-26], making the rapid muscle fatigue induced by NMES a continuing issue $[27,28]$. A clinical issue is therefore presented since higher volumes of strength training, in comparison to lower volumes, are known to promote greater health and functional adaptations, particularly in older adults and in clinical populations such as people with diabetes type $2[29,30]$. For these reasons, early muscle fatigue represents a problem and, if suboptimal stimulation parameters are used and NMES training elicits a rapid muscle fatigue, may prevent the delivery of the ideal dose of training to realise optimum musculoskeletal adaptations in paralysed muscles. Nonetheless, NMES as a strength training modality has previously been shown to stimulate quadriceps muscle hypertrophy [31-34], improved skeletal muscle oxidative capacity [35] and to improve lean muscle mass [33] and bone adaptations in people with SCI [36-38]. Yet, the use of NMES as high-intensity strength training mode has not been extensively investigated and, due to the limited evidence supporting its use for increasing muscle strength $[39,40]$ is not commonly used in clinical practice.

One promising method to enhance force production whilst minimising muscle fatigue is the application of tendon vibration $[41,42]$. Tendon vibration may generate trains of Ia-afferent signals to the spinal cord that induce a progressive excitation of homonymous motor neurones and promote the development of persistent inward calcium $\left(\mathrm{Ca}^{2+}\right)$ or sodium $\left(\mathrm{Na}^{+}\right)$currents at their dendritic trees [43-45]. Thus, this method could amplify and prolong the synaptic input and create a sustained depolarisation leading to an increased recruitment of motor units (i.e. self-sustained firing) and increase muscle force production, especially when coupled with wide-pulse width (e.g. $1000 \mu \mathrm{s})$ NMES [46, 47]. Importantly, however, vibration tends to preferentially excite low-threshold (i.e. fatigue resistant) motor units [48], which exhibit significant endurance and are therefore likely to contribute to a great total muscular work before fatigue is induced. In previous studies the positive effects of vibration were observed in able-bodied populations during wide-pulse width NMES applied both at relatively low ( 5\% MVC force) [46] and high ( 20\% MVC force) [47] levels of muscle force production, suggesting that vibration may augment muscle contraction force and allow a greater total muscular effort before fatigue during wide-pulse width NMES even when relatively high contraction intensities are used, such as those required for strength training. However, it is unknown whether the imposition of tendon vibration onto wide-pulse width NMES promotes such benefits in people with SCI. In fact, it is also not known whether tendon vibration might elicit an additional excitation of the motor neuron pool through the same (central) pathways as wide-pulse width NMES $[43-45,49]$ and therefore instead promote a greater muscle fatigue in partially or completely paralysed muscles.

In a previous study [50], patellar tendon vibration during wide-pulse width NMES in able-bodied individuals resulted in an increased total muscle work performed in "positive responders" only, but a reduced total work in others. Nonetheless, within the whole study cohort the 
application of patellar tendon vibration tended to minimise voluntary muscle fatigue caused by the NMES [50], and would thus have allowed the study participants to immediately perform other physical activities without a notable negative impact on performance. Thus, it is of great interest to determine whether similar effects would be obtained in people with SCI. The purpose of the present study, therefore, was to determine whether patellar tendon vibration superimposed onto wide-pulse width NMES under standard clinical conditions elicits a greater peak muscle force with less muscle fatigue (i.e. a greater total impulse) when compared to NMES applied without patellar tendon vibration in people with SCI. We hypothesised that patellar tendon vibration superimposed onto wide-pulse width NMES would elicit a greater peak muscle force with less muscle fatigue (i.e. a greater total impulse) that NMES applied without patellar tendon vibration in people with SCI.

\section{Methods}

\section{Subjects}

Nine subjects with SCI (3 females, 6 males) were recruited from the Spinal Cord Injuries Australia (SCIA) Activitybased therapy exercise program "NeuroMoves" and the Perth community (mean \pm SD, age: $39.4 \pm 10.6$ y; height: $176.2 \pm 9.7 \mathrm{~cm}$; body mass $80.6 \pm 9.6 \mathrm{~kg}$ ). Four subjects were classified as complete spinal cord injury (AIS A) and five were classified as incomplete (AIS B-D). All subjects were recruited by word of mouth or email and had a SCI of more than 6 months. Prior to the study, subjects were given detailed information about the procedures and the risks of participating in the study, and they all signed a written consent form. Subjects completed the Physical Activity Readiness Questionnaire (PAR-Q) to ensure safe exercise participation and refrained from vigorous exercise (48 h) and alcohol $(24 \mathrm{~h})$ and stimulant consumption (e.g. caffeine, energy drinks for $6 \mathrm{~h}$ ) prior to testing.
Participants included in this study had medical clearance and were fit and medically stable to participate in this study. Participants were asked to replicate the same physical routine for each session. Sessions were completed at the same time of the day and under the same experimental conditions. This research study was approved by the Edith Cowan University Human Ethics Committee (reference number: 11,623). Subject characteristics are detailed in Table 1.

\section{Procedures}

The procedures used in this study followed the methodology described in a previous study in healthy individuals [50], but was adapted to people with SCI accordingly. All subjects attended the Neuromuscular Physiology Laboratory at Edith Cowan University on three occasions on different days (1 day per week for the duration of 4 weeks) with a minimum of 7 days between sessions. One week before starting data collection, the subjects attended a full familiarisation session where the NMES protocol was applied to the dominant quadriceps femoris with and without simultaneous patellar tendon vibration. Each subject received $1 \mathrm{~min}$ of tendon vibration and $3 \mathrm{~min}$ of NMES to ensure they could tolerate the intervention; all subjects tolerated the NMES and tendon vibration protocols well. The subsequent two sessions were used to complete the following two experimental protocols in a random order without replication: 1) NMES only (STIM); and 2) NMES superimposed onto tendon vibration (STIM+Vib).

In familiarisation and experimental sessions, the subjects were asked to produce a voluntary knee extension contraction of approximately $50 \%$ of perceived maximal voluntary exertion while seated on an isokinetic dynamometer. If any voluntary contractions were visualised and recorded, then a standardised warm-up protocol was performed, consisting of isometric knee extensions

Table 1 Subject characteristics. Subject levels of injury, completeness of lesion, time since injury, AIS scale score, medication type and response to tendon vibration (i.e. whether a tendon vibration reflex response is detectable). Complete (C) lesion means no voluntary muscle contraction below the level of injury. Incomplete (I) lesion means some voluntary muscle contraction below the level of injury [71]

\begin{tabular}{|c|c|c|c|c|c|c|c|}
\hline Subject & Level of injury & Complete (C)/Incomplete (I) & Time since injury (y) & AIS & Medication & $\begin{array}{l}\text { Positive responder } \\
\text { to tendon vibration }\end{array}$ & $\begin{array}{l}\text { Negative responde } \\
\text { to tendon vibration }\end{array}$ \\
\hline A & $\mathrm{T}_{7}$ & I & 3 & B & Baclofen & & $x$ \\
\hline B & $\mathrm{T}_{6}$ & C & 6 & A & Oxybutin & & $x$ \\
\hline C & $T_{6}$ & C & 5 & A & Baclofen & & $x$ \\
\hline D & $\mathrm{C}_{6}-\mathrm{C}_{7}$ & C & 4 & A & Baclofen & & $x$ \\
\hline E & $\mathrm{T}_{12}$ & । & 2 & D & Baclofen & & $x$ \\
\hline $\mathrm{F}$ & $C_{7}$ & C & 1 & A & Baclofen & $x$ & \\
\hline G & $T_{5}$ & I & 20 & $\mathrm{D}$ & Baclofen & $x$ & \\
\hline H & $T_{3}$ & I & 2 & B & Baclofen & $x$ & \\
\hline I & $L_{3}$ & I & 4 & $\mathrm{D}$ & N/A & $x$ & \\
\hline
\end{tabular}


at $30 \%, 50 \%, 70 \%$ and $90 \%$ of perceived maximal effort before performing a series of three knee extension MVICs. However, if no voluntary contraction was recorded then three attempts of maximal voluntary contractions (MVICs) were instructed without warm-up efforts. The subjects were seated with hip and knee joint angles of $85^{\circ}$ and $90^{\circ}$, respectively $\left(0^{\circ}=\right.$ full knee extension), with the thigh and trunk secured to the dynamometer chair and the knee joint aligned with the center of rotation of the dynamometer. All subjects were instructed to produce a force against the dynamometer arm by extending the knee as fast and hard as possible for $3 \mathrm{~s}$, and verbal encouragement and visual feedback were provided during all MVICs irrespective of the subject's ability to voluntarily activate their lower limbs. This method was implemented to be consistent with the procedures among all subjects.

\section{Electrical stimulation and tendon vibration protocols}

NMES was delivered by a high-voltage constant-current electrical stimulator (400 V, DS7A, Digitimer Ltd., Welwyn Garden City, UK) through four self-adhesive stimulation electrodes (Axelgaard, PALS, USA) placed over the rectus femoris (RF), vastus lateralis (VL), and vastus medialis (VM); two $5 \times 10 \mathrm{~cm}$ electrodes were placed over RF and one $5 \times 5 \mathrm{~cm}$ electrode was placed on each of the VM and VL, approximately at their motor points. The electrodes were then moved if necessary to elicit the greatest twitch response with low stimulation intensity in every session [51]. The electrode positions were marked on a plastic sheet for each subject and indelible ink was used to mark these positions on the skin to ensure identical electrode placement at subsequent sessions.

To habituate the subjects to the electrical stimulations- and after the attempts of MVCIs, two electrical square-wave stimuli (1000 $\mu$ s square-wave pulses separated by $5 \mathrm{~ms}$ ) were delivered to the dominant leg (determined in familiarisation session using NMES) every $20 \mathrm{~s}$ while the stimulation current was increased from 30 to $99 \mathrm{~mA}$ in $10-\mathrm{mA}$ increments until a plateau in the maximum peak twitch torque was observed. This was defined as maximal peak twitch torque $\left(\tau_{t w, p}\right)$ and was used as the "target torque". A second, submaximal peak twitch torque $\left(\tau_{\mathrm{tw}, \mathrm{sub}}\right)$ recording was obtained at $40 \mathrm{~mA}$ and retained for analysis of changes in submaximal torque, to assess the ability of the muscle to contract under submaximal conditions, which are often used in a clinical context. Subsequently, a maximum of three trains of NMES (described below) were performed at different stimulation current intensities until reaching the closest value to the target torque. This procedure was replicated in all participants regardless of their response to the imposed current. All participants in this study showed an increase in evoked torque when increasing the current from 30 to $99 \mathrm{~mA}$ (maximum output allowed by the electrical stimulator) and the level of evoked torque was set equal or close to the maximal peak twitch torque $\left(\tau_{\mathrm{tw}, \mathrm{p}}\right)$.

The NMES protocol consisted of repeated $30-\mathrm{Hz}$ trains of 58 wide-pulse width $(1000 \mu \mathrm{s})$ symmetric biphasic pulses (0.033-s inter-pulse interval), where a single train duration was $2 \mathrm{~s}$ and the inter-train interval was 2 s (i.e. 2-s on and 2-s off). The level of evoked torque was set equal or close to the maximal peak twitch torque $\left(\tau_{\mathrm{tw}, \mathrm{p}}\right)$, which should be equivalent to $\sim 20 \%$ of MVIC.

Patellar tendon vibration was applied using a vibration device (Deep Muscle Stimulator, Las Vegas, NV, USA), which mechanically vibrated the tendon at $55 \mathrm{Ha}$ and amplitude of $7 \mathrm{~mm}$ (confirmed through direct measurement of high-speed video). The two test conditions were:

STIM: Electrically-evoked muscle contractions produced by delivering the NMES protocol until torque was reduced to $\leq 50 \%$ of the target torque (i.e. $\tau_{t w, p}$ ) in one electrically-evoked contraction, which was defined as "target fatigue".

STIM + Vib: Electrically-evoked contractions delivered as in STIM, but superimposed with patellar tendon vibration which was applied for at least $5 \mathrm{~s}$ before NMES and after target fatigue was reached.

For a graphical representation of the STIM and STIM + Vib protocols please refer to Fig. 1.

\section{Data collection and analysis \\ Peak evoked torque, torque-time integral and number of contractions}

Two measures of peak-twitch evoked torque were analysed: one submaximal ( $\left.\tau_{\mathrm{tw}, \mathrm{sub}}\right)$ evoked at a $40-\mathrm{mA}$ current, and one maximal $\left(\tau_{t w, p}\right)$ obtained from the peak of the torque-current relationship. These were obtained both before and after the NMES protocols were delivered. The total torque-time integral (TTI) was used to provide a measure of the total exercise stimulus received by the muscle in each condition. TTI was calculated as the product of torque and time calculated from the onset of the first stimulation train (STIM) or vibration onset $(\mathrm{STIM}+\mathrm{Vib})$ to the end of the final evoked contraction when "target fatigue" was reached (as defined in Procedures). Peak evoked torque was defined as the highest torque value obtained after the onset of the first stimulation train for both STIM and STIM+Vib. TTI and peak evoked torque were compared between STIM and STIM+Vib. The post-study data analysis revealed that some subjects responded with a greater TTI 


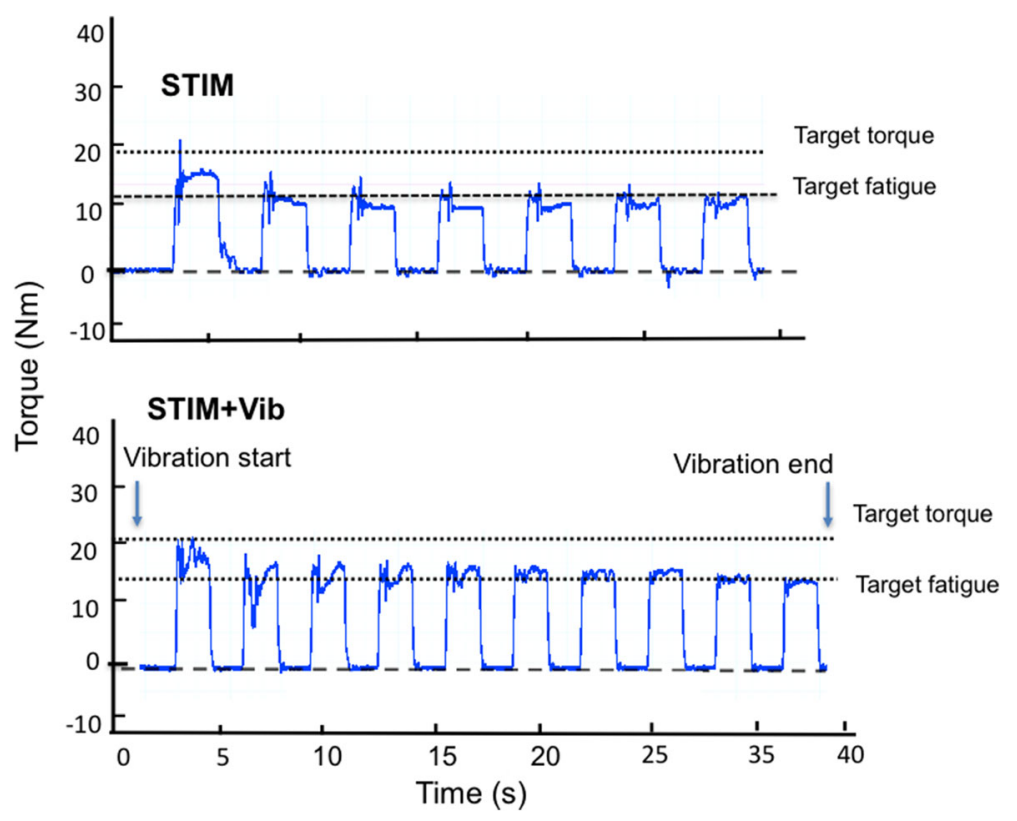

Fig. 1 Graphical representation of the STIM and STIM+Vib. STIM and STIM+Vib protocols in one of the participants can be observed in this graph. Blue traces refer to the torque forces evoked by the NMES protocols

after STIM+Vib than STIM (i.e. positive responders to patellar tendon vibration) whilst others showed no difference or a lower TTI after STIM+Vib than STIM (i.e. negative responders to patellar tendon vibration), as described in Results. Therefore, a second analysis was performed after separating subjects into positive and negative responders to patellar tendon vibration (described in Procedures). Positive and negative responders were identified not only during the training sessions but also during pilot testing sessions where different NMES protocols with superimposed patellar tendon vibration were utilised. Although the pilot data are not presented in the present article, this information reinforced the identification of positive and negative responders to patellar tendon vibration during at least one pilot session and provided confidence in the interpretation of results. The total number of contractions was measured as the number of contractions from the beginning of the first evoked contraction reaching the target torque until the last contraction when reaching $50 \%$ of the target torque ("target fatigue").

\section{Statistical analysis}

Two-way repeated measures analysis of variance (ANOVA) was used to compare changes in all variables between conditions (STIM, STIM+Vib) over time (PRE, POST). A Wilcoxon test was conducted to compare STIM, STIM+Vib between PRE-and POST in positive and negative responders to patellar tendon vibration whilst using assessments at PRE as the covariates. Pairwise $t$-tests were performed when significant interaction effects were found. A chi- square test for independence was used to assess whether an association existed between subjects with complete and incomplete SCI and the likelihood of being a positive or negative responders. Statistical significance was set at an alpha level of $p \leq$ 0.05 and values are reported as mean \pm SD.

\section{Results}

Peak evoked torque, torque-time integral (TTI) and total number of contractions

No statistical differences in peak evoked torque $(p=$ 0.43; Fig. 2a), torque-time integral (TTI; $p=0.39$; Fig. $2 \mathrm{~b})$ or total number of contractions $(p=0.78)$ were observed between STIM and STIM+Vib. Nonetheless, the response to STIM+Vib (based on TTI) was clearly greater than in STIM in some subjects (40\% of sample) but lesser (or negative) in others. Thus, an additional comparative analysis of positive versus negative responders to patellar tendon vibration was undertaken, where positive responders to patellar tendon vibration were defined as those subjects who responded with a greater TTI in STIM+Vib when compared to STIM. This analysis revealed no statistical difference in TTI between STIM and STIM+Vib for positive or negative responders (Fig. 3), or a difference between STIM and STIM+Vib for the whole cohort collectively. However, the between-condition difference was dissimilar between the responder groups when using $\tau_{\mathrm{tw}, \mathrm{p}}$ at PRE for both conditions as a covariate $(p=0.02)$; TTI was $59.2 \pm$ $15.8 \%$ greater in STIM+Vib than STIM in positive responders to patellar tendon vibration $(p=0.13)$ but 31.3 

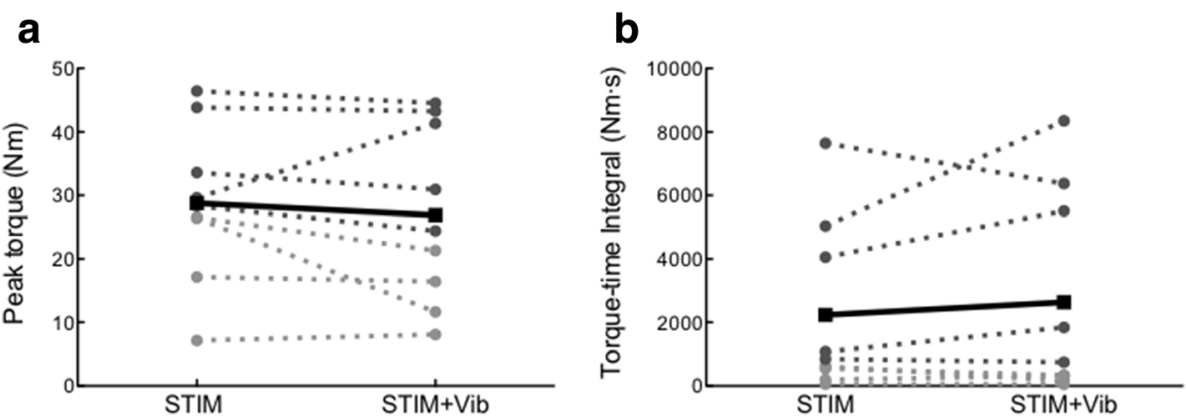

Fig. 2 Peak torque and Torque-Time Integral (TTI) in STIM and STIM+Vib conditions. a Peak torque production (Nm) in STIM and STIM+Vib conditions for all subjects. No statistically significant differences were found between the two conditions $\mathbf{b}$ Torque-time integral (TTI; Nm.S) in STIM and STIM+Vib conditions for all subjects. No statistically significant differences were found between the two conditions. Grey dashed lines represent individual subjects and the black solid line represents the group mean. Darker grey dots indicate subjects with incomplete spinal cord injury (SCI) and light grey dots indicate subjects with complete SCI

$\pm 25.7 \%$ less in STIM+Vib than STIM in negative responders $(p=0.14)$, as shown in Fig. 3. There was also no clear effect of completeness of lesion (complete or incomplete SCI) on the likelihood of being a positive or negative responder to tendon vibration $\left(\chi^{2}(1, n=9)=\right.$ 1.10, $p=0.70)$. Also, no significant differences were found between the conditions (STIM and STIM+Vib) for total number of contractions, despite a trend being observed; the mean total number of contractions for positive responders to tendon vibration for STIM was $56.5 \pm 60.5$ and for STIM+Vib was $70.2 \pm 68.9$, whilst the means for negative responders to tendon vibration were

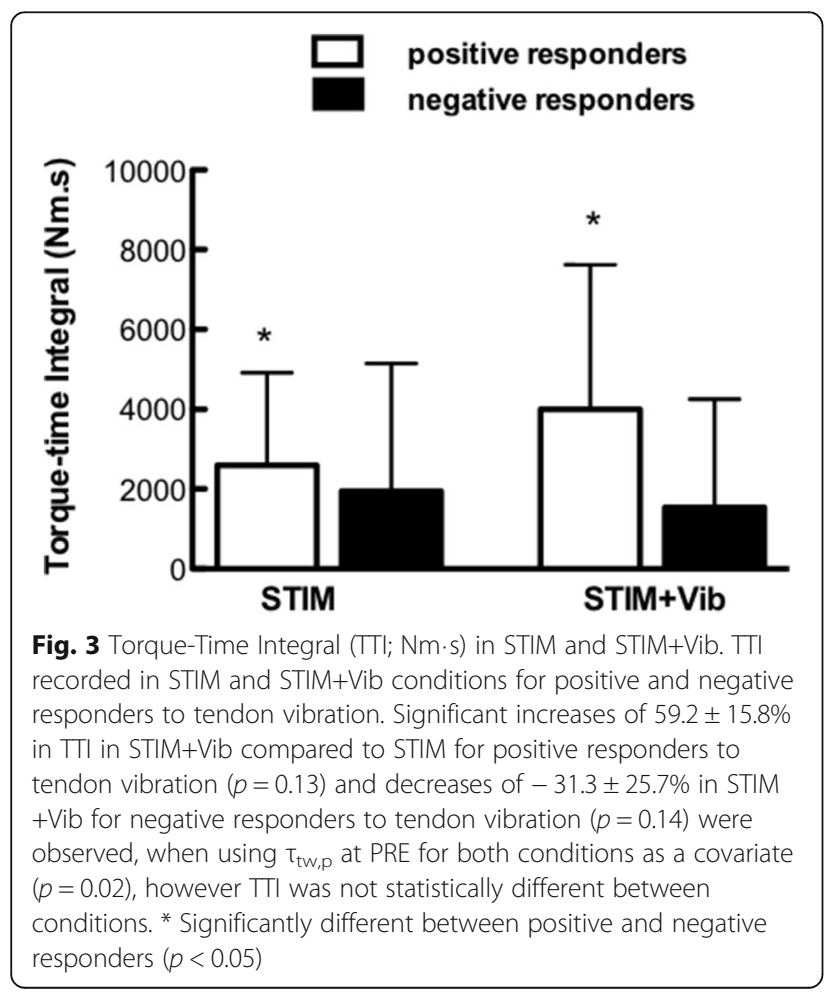

$41.6 \pm 37.4$ for STIM and $33.8 \pm 30.9$ for STIM+Vib (figure not shown).

Muscle force measures: Maximal evoked force $\left(\tau_{t w, p}\right)$ and submaximal evoked force $\left(\tau_{t w, s u b}\right)$

There was a significant effect of time, as the relative maximal force $\left(\tau_{\mathrm{tw}, \mathrm{p}} ; p=0.00\right)$ and submaximal force ( $\left.\tau_{\mathrm{tw}, \mathrm{sub}} ; p=0.00\right)$ decreased from PRE to POST in both conditions (STIM and STIM+Vib). However, a similar pattern was observed in STIM+Vib and STIM conditions, as there were no significant effects of condition $\left(\tau_{\mathrm{tw}, \mathrm{p}}: p=0.21 ; \tau_{\mathrm{tw}, \mathrm{sub}}: p=0.13\right)$ and no significant condition $\times$ time interaction $\left(\tau_{\mathrm{tw}, \mathrm{p}}: p=0.98 ; \tau_{\mathrm{tw}, \mathrm{sub}}: p=0.77\right)$. Submaximal twitch torque $\left(\tau_{\mathrm{tw}, \mathrm{sub}}\right)$ declined by $40.4 \pm$ $4.7 \%$ and maximal force $\left(\tau_{t w, p}\right)$ declined by $27.0 \pm 5.0 \%$ of baseline in STIM, whilst $\tau_{\mathrm{tw} \text {,sub }}$ declined by $45.0 \pm 4.2 \%$ and $\tau_{\mathrm{tw}, \mathrm{p}}$ declined by $30.6 \pm 5.0 \%$ of baseline on STIM +Vib (Fig. 4).

\section{Discussion}

The purpose of this study was to determine whether patellar tendon vibration superimposed onto wide-pulse width NMES would elicit greater peak muscle force production and/or induce less muscle fatigue (i.e. increase the total impulse before fatigue) than NMES alone in people with spinal cord injury (SCI). The main finding was that the torque-time integral (TTI) measured at the point of fatigue (i.e. $50 \%$ of initial evoked torque) was not statistically different between STIM and STIM+Vib conditions, although a significantly greater TTI was found in four (of nine) "positive" responders to patellar tendon vibration $(59.2 \pm 15.8 \%)$. Thus, in four of the present participants, the addition of the patellar tendon vibration allowed for a greater total muscular work to be performed, but a lower total muscular work was completed in the other five subjects $(-31.3 \pm 25.7 \%)$. However, these changes were not statistically different 


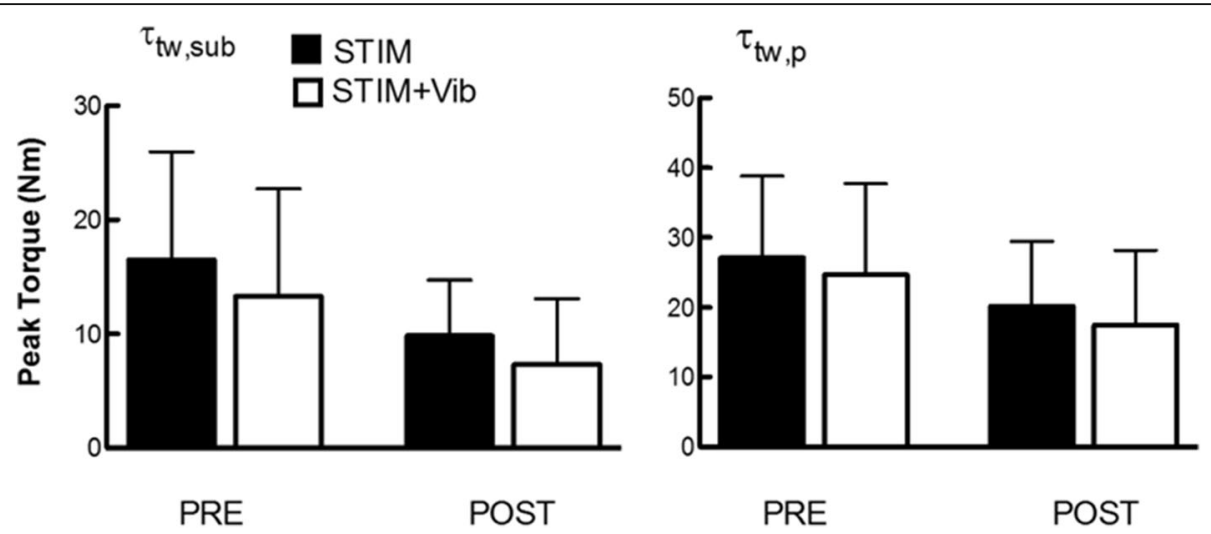

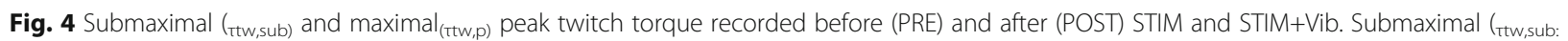

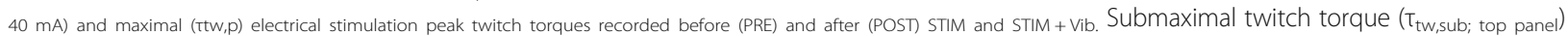

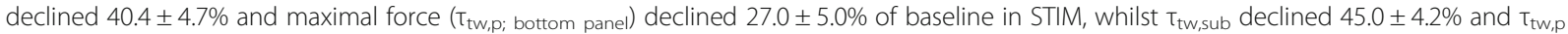
declined $30.6 \pm 5.0 \%$ of baseline in STIM+Vib. However, no statistically significant differences were found between the two conditions

between conditions. Moreover, as observed from Fig. 2, there was no evidence that completeness of lesion (complete vs. incomplete SCI) was a factor influencing the likelihood of being a responder to patellar tendon vibration. Therefore, whilst the imposition of patellar tendon vibration during wide-pulse width NMES may allow for a greater impulse to be provided before fatigue in some individuals, it is not apparently clear who might benefit from the application of patellar tendon vibration in people with SCI based on our results.

The use of patellar tendon vibration superimposed onto wide-pulse width NMES was hypothesised to allow for a greater training impulse prior to fatigue, which might be of practical significance since it might evoke greater chronic increases in muscle strength and mass as well as improve muscle performance after a period of training $[36,52]$. However, this appears to be the case only in some individuals who show a positive response to patellar tendon vibration. The ability to produce greater forces after a period of training may allow for higher volumes of muscle work to be performed during a physical rehabilitation session and thus generate early improvements in physical performance and physical health benefits in people with $\mathrm{SCI}$; this hypothesis should thus be tested using a longitudinal study design. A reflex response induced by patellar tendon vibration may facilitate the initiation, maintenance and strength of any residual voluntary contraction in people with incomplete SCI. The use of patellar tendon vibration in those with a complete lesion can help to regulate the reflex response, which is highly altered after a complete SCI, by stimulating reflex pathways. Previous research has shown that somatosensory information continues to flow in a modified manner after a complete SCI and that there is a potential to generate motor patterns if this somatosensory input is stimulated by the use of methods such as NMES and tendon vibration $[53,54]$. The mechanisms of action of tendon vibration relate to the tonic vibration reflex (TVR), elicited by the application of tendon vibration, which may potentially help recruit more motor units and thus increase muscle force production when used in combination with NMES [46]. Greater muscular forces elicited by the tendon vibration when superimposed to NMES were found in previous studies in able-bodied people [46] and were attributed to the generation of persistent inward currents, recruiting higher-threshold motor units through reflexive pathways and increasing muscle force production $[55,56]$. On the other hand, the reduced training impulse prior to fatigue observed in the negative responders to patellar tendon vibration suggested that patellar tendon vibration may be detrimental for some people and that in these people the use of (wide-pulse width) NMES alone may be more beneficial. This response observed in negative responders to patellar tendon vibration may speculatively be caused by the low-to-moderate frequency $(55 \mathrm{~Hz})$ vibration resulting in a stimulation of Golgi tendon organs, which can cause autogenic inhibition and potentially decrease the motor output [57]. Therefore, the present, individual-specific results cannot confirm the hypothesis that superimposing patellar tendon vibration onto wide-pulse width NMES would elicit an additional greater peak muscle force with less muscle fatigue (i.e. a greater total impulse) when compared to NMES applied without patellar tendon vibration in most people with a SCI.

These variable responses to patellar tendon vibration may also be explained by the highly individual functional deficits found between individuals with incomplete SCI, where the transmission of the sensory-motor information is altered at the synaptic level $[58,59]$ and there is a muscle spindle afferent dysfunction in both complete 
and incomplete lesions to the spinal cord [58, 59]. A similar inter-individual variability in response to widepulse width NMES has also been suggested to originate from a difference in monoamine levels between individuals [43] and could be one reason for the different response to patellar tendon vibration superimposed onto wide-pulse width NMES observed in the current study. This difference in monoamine levels could be exacerbated by the altered neuromuscular system in people with spinal cord injuries [60], such as changes in the excitability of the motor neurone [61], fibre type transformation towards fast-fatigable [60] and high levels of muscle atrophy [62]. Another factor that could have decreased the stretch reflex and thus prevented the formation of PICs is the use of antispasmodic medications, such as Baclofen [63]. On the other hand, the increased TTI in positive responders to patellar tendon vibration might be attributable to the development of tonic vibration reflexes (TVR) which increase muscle force contributions between the evoked muscular contractions [47, 64]. However, the activation of already hyper-excitable sensory pathways by the use of patellar tendon vibration in people suffering from a spinal cord injury may have either triggered episodes of intrinsic phasic spasticity in some participants [65], whilst attenuated spasticity symptoms in others [66], and thus may have increased the variability in the response to wide-pulse width NMES and patellar tendon vibration observed in the present study. These hypotheses will need to be explored in further studies investigating explicitly the pathophysiological responses (i.e. spasticity) of paralysed muscles to tendon vibration superimposed onto wide-pulse width NMES.

Another important finding of the current study was that the decline in evoked muscle force was not attenuated by the application of patellar tendon vibration. Muscle fatigue experienced after both STIM and STIM $+\mathrm{Vib}$ could be attributed to the "peripheral fatigue" (i.e. contractile alterations) induced by NMES, due to the repeated activation of the same muscle fibres, especially due to the missing sensory feedback from the muscles to the spinal cord to prevent failure after a SCI [67-69]. It may also be possible that patellar tendon vibration activated not only excitatory but also inhibitory interneurons and thus negated the possible positive effects of patellar tendon vibration on muscle fatigue [70]. Accordingly, results from this study are inconclusive regarding the effects of superimposing patellar tendon vibration onto wide-pulse width NMES in people with SCI and future research studies are needed to investigate the mechanisms of action of tendon vibration in chronically paralysed muscles. Moreover, the present results remain to be verified in future studies in a larger cohort of people with SCI, whilst also considering the potentiallyconfounding factors of age, level of injury, AIS classification (complete vs. incomplete), time since injury, spasticity and medication use, which may impact in the measured outcomes.

\section{Conclusion}

The imposition of patellar tendon vibration onto moderate-frequency, wide-pulse width NMES may allow for a greater amount of muscular work (muscle force production) to be completed before fatigue in a proportion of participants (i.e. positive responders to tendon vibration) with SCI. However, a lesser response may also be elicited in participants who respond negatively to patellar tendon vibration (60\% in the current study) and thus, for those cases, wide-pulse width NMES alone may provide greater benefits. The use of patellar tendon vibration superimposed onto wide-pulse width NMES did not minimise the (peripheral) fatigue elicited by the training session. Based on the present results there is insufficient evidence to conclude that patellar tendon vibration improves muscle force production during the use of wide-pulse width NMES in people with SCI. In the clinic, it is suggested that people with SCI are assessed for their response to patellar tendon vibration in order that the appropriate protocol is implemented. Nonetheless, replication of these findings is required before clear decisions as to whether to implement the strategy in clinical practice can be made.

\section{Abbreviations \\ $\tau_{\mathrm{tw}, \mathrm{p}}$ : Maximal evoked torque (peak twitch torque); $\tau_{\mathrm{tw}, \text { sub: }}$ Submaximal evoked torque (peak twitch torque); AIS: American Spinal Injury Association Impairment Scale; FES: Functional electrical stimulation; MVC: Maximal voluntary contraction; MVIC: Maximal voluntary isometric contraction; NMES: Neuromuscular electrical stimulation; QoL: Quality of life; RF: Rectus femoris; SCI: Spinal cord injury; STIM: Neuromuscular electrical stimulation protocol; STIM+Vib: Neuromuscular electrical stimulation superimposed onto tendon vibration protocol; TTI: Torque-time integral; VL: Vastus lateralis; VM: Vastus medialis}

\section{Acknowledgements}

Not applicable.

\section{Funding}

Spinal Cord Injuries Australia (SCIA) Collaborative Research Program grant to Robert Newton.

\section{Availability of data and materials}

The datasets used and analysed during the current study are available from the corresponding author on reasonable request.

\section{Authors' contributions}

$V B, A B, R N$, GT made substantial contributions to conception and design of this study. VB, AB, AV, GT, TP made substantial contributions to acquisition of data, analysis and interpretation of data. All authors participated in drafting the article or revising it critically for important intellectual content. All authors gave final approval of the version to be submitted and any revised version.

\section{Ethics approval and consent to participate}

Prior to the study, subjects were given detailed information about the procedures and the risks of participating in the study, and they all signed a written consent form. This research study was approved by the Edith Cowan University Human Ethics Committee (reference number: 11623). 


\section{Consent for publication}

Not applicable.

\section{Competing interests}

The authors declare that they have no competing interests.

\section{Publisher's Note}

Springer Nature remains neutral with regard to jurisdictional claims in published maps and institutional affiliations.

\section{Author details}

'Department of Exercise and Health Sciences, School of Health, Medical and Applied Sciences, Central Queensland University, Building 34.1.02, Bruce Highway, North Rockhampton, Qld 4702, Australia. ${ }^{2}$ Exercise Medicine Research Clinic, Edith Cowan University, Perth, Australia. ${ }^{3}$ Centre for Sports and Exercise Science, School of Medical and Health Sciences, Edith Cowan University, Joondalup, Australia. ${ }^{4} U Q$ Centre for Clinical Research, The University of Queensland, Brisbane, Australia. ${ }^{5}$ School of Exercise and Nutrition Sciences, Institute of Health and Biomedical Innovation, Queensland University of Technology (QUT), Brisbane, Australia.

‘UDF-University Centre, Brasilia, Brazil.

\section{Received: 5 September 2017 Accepted: 2 February 2018} Published online: 13 February 2018

\section{References}

1. Tewarie RDS, Hurtado A, Bartels RHMA, Grotenhuis JA, Oudega M. A clinical perspective of spinal cord injury. Neurorehabil. 2010;27(2):129-39.

2. Oyster ML, Karmarkar AM, Patrick M, Read MS, Nicolini L, Boninger ML. Investigation of factors associated with manual wheelchair mobility in persons with spinal cord injury. Arch of Phys Med and Rehab. 2011;92(3): 484-90.

3. Hosseini SM, Oyster ML, Kirby RL, Harrington AL, Boninger ML. Manual wheelchair skills capacity predicts quality of life and community integration in persons with spinal cord injury. Arch of Phys Med and Rehab. 2012; 93(12):2237-43.

4. Schaap LA, Pluijm SM, Deeg DJ, Harris TB, Kritchevsky SB, Newman AB, et al. Higher inflammatory marker levels in older persons: associations with 5-year change in muscle mass and muscle strength. J Gerontol A Biol Sci Med Sci. 2009;64((11):1183-9.

5. Srikanthan P, Karlamangla AS. Muscle mass index as a predictor of longevity in older adults. Am J Med. 2014;127(6):547-53.

6. Orlando G, Balducci S, Bazzucchi I, Pugliese G, Sacchetti M. Neuromuscular dysfunction in type 2 diabetes: underlying mechanisms and effect of resistance training. Diabetes-Metab Res Rev. 2016;32(1):40-50.

7. Andrade SD, da Silva JN. The effects of resistance training in osteoporosis: a systematic review. RBNE. 2015;9(50):144-9.

8. Clark JE, Goon DT. The role of resistance training for treatment of obesity related health issues and for changing health status of the individual who is overfat or obese: a review. J Sports Med Phys Fitness. 2015;55(3):205-22.

9. Caserotti P, Aagaard P, Larsen JB, Puggaard L. Explosive heavy-resistance training in old and very old adults: changes in rapid muscle force, strength and power. Scand J Med Sci Sports. 2008:18(6):773-82.

10. Barbeau H, Ladouceur M, Mirbagheri MM, Kearney RE. The effect of locomotor training combined with functional electrical stimulation in chronic spinal cord injured subjects: walking and reflex studies. Brain Res. 2002;40(1-3):274-91

11. Harvey LA, Fornusek C, Bowden JL, Pontifex N, Glinsky J, Middleton JW, et al. Electrical stimulation plus progressive resistance training for leg strength in spinal cord injury: a randomized controlled trial. Spinal Cord. 2010;48(7): 570-5.

12. Thrasher TA, Ward JS, Fisher S. Strength and endurance adaptations to functional electrical stimulation leg cycle ergometry in spinal cord injury. Neurorehabil. 2013:33(1):133-8.

13. American College of Sports Medicine. Progression models in resistance training for healthy adults. Med Sci Sports Exerc. 2009:41(3):687-708.

14. Ahtiainen JP, Pakarinen A, Alen M, Kraemer WJ, Hakkinen K. Muscle hypertrophy, hormonal adaptations and strength development during strength training in strength-trained and untrained men. Eur J Appl Physiol. 2003:89(6):555-63.

15. Enoka R. Neuromechanics of human movement 2002.
16. Hortobagyi T, Maffiuletti NA. Neural adaptations to electrical stimulation strength training. Eur J Appl Physiol. 2011:111(10):2439-49.

17. Alon G. Guest editorial - Use of neuromuscular electrical stimulation in neureorehabilitation: A challenge to all. J Rehabil Res Dev. 2003;40(6):IX-XII.

18. Hillegass EA, Dudley GA. Surface electrical stimulation of skeletal muscle after spinal cord injury. Spinal Cord. 1999;37(4):251-7.

19. Gregory CM, Recruitment BCS. Patterns in human skeletal muscle during electrical stimulation. Phys Ther. 2005:85(4):358-64.

20. Gorgey AS, Mahoney E, Kendall T, Dudley GA. Effects of neuromuscular electrical stimulation parameters on specific tension. Eur J Appl Physiol. 2006;97(6):737-44.

21. Ibitoye MO, Hamzaid NA, Hasnan N, Abdul Wahab AK, Davis GM. Strategies for rapid muscle fatigue reduction during fes exercise in individuals with spinal cord injury: a systematic review. PLoS One. 2016;11(2):e0149024.

22. Bickel CS, Gregory CM, Dean JC. Motor unit recruitment during neuromuscular electrical stimulation: a critical appraisal. Eur J Appl Physiol. 2011:111(10):2399-407.

23. Bickel CS, Slade JM, VanHiel LR, Warren GL, Dudley GA. Variable-frequencytrain stimulation of skeletal muscle after spinal cord injury. J Rehabil Res Dev. 2004;41(1):33-40

24. Adams GR, Harris RT, Woodard D, Dudley GA. Mapping of electrical muscle stimulation using MRI. J Appl Physiol. 1993;74(2):532-7.

25. Burnham R, Martin T, Stein R, Bell G, MacLean I, Steadward R. Skeletal muscle fibre type transformation following spinal cord injury. Spinal Cord. 1997;35(2):86-91.

26. Pelletier CA, Hicks AL. Muscle fatigue characteristics in paralyzed muscle after spinal cord injury. Spinal Cord. 2011;49(1):125-30.

27. Karu ZZ, Durfee WK, Barzilai AM. Reducing muscle fatigue in fes applications by stimulating with N-let pulse trains. IEEE Trans Biomed Eng. 1995:42(8):809-17.

28. Gorgey AS, Poarch HJ, Dolbow DR, Castillo T, Gater DR. Effect of adjusting pulse durations of functional electrical stimulation cycling on energy expenditure and fatigue after spinal cord injury. J Rehab Res Dev. 2014;51(9):1455-68.

29. Borde R, Hortobagyi T, Granacher U. Dose-response relationships of resistance training in healthy old adults: a systematic review and metaanalysis. Sports Med. 2015;45(12):1693-720.

30. Schoenfeld BJ, Ogborn D, Krieger JW. Dose-response relationship between weekly resistance training volume and increases in muscle mass: a systematic review and meta-analysis. J Sports Sci. 2017;35(11):1073-82.

31. Bickel CS, Yarar-Fisher C, Mahoney ET, McCully KK. Neuromuscular electrical stimulation-induced resistance training after SCl: a review of the Dudley protocol. Top Spinal Cord Inj Rehabil. 2015;21(4):294-302.

32. Dudley GA, Castro MJ, Rogers S, Apple DF. A simple means of increasing muscle size after spinal cord injury: a pilot study. Eur J Appl Physiol Occup Physiol. 1999;80(4):394-6

33. Gorgey AS, Mather KJ, Cupp HR, Gater DR. Effects of resistance training on adiposity and metabolism after spinal cord injury. Med Sci Sports Exerc. 2012 44(1):165-74.

34. Mahoney ET, Bickel CS, Elder C, Black C, Slade JM, Apple D, et al. Changes in skeletal muscle size and glucose tolerance with electrically stimulated resistance training in subjects with chronic spinal cord injury. Arch Phys Med Rehabil. 2005:86(7):1502-4.

35. Erickson ML, Ryan TE, Backus D, McCully KK. Endurance neuromuscular electrical stimulation training improves skeletal muscle oxidative capacity in individuals with motor-complete spinal cord injury. Muscle Nerve. 2017;55(5):669-75.

36. Dudley-Javoroski S. Muscle and bone plasticity after spinal cord injury: review of adaptations to disuse and to electrical muscle stimulation. J Rehabil Res Dev. 2008;45(2):283-96.

37. Hangartner TN, Rodgers MM, Glaser RM, Barre PS. Tibial bone density loss in spinal cord injured patients: effects of FES exercise. J Rehabil Res Dev. 1994; 31(1):50-61.

38. Shields RK, Dudley-Javoroski S, Law LA. Electrically induced muscle contractions influence bone density decline after spinal cord injury. Spine. 2006;31(5):548-53.

39. Glinsky J, Harvey L, Van Es P. Efficacy of electrical stimulation to increase muscle strength in people with neurological conditions: a systematic review. Physiother Res Int. 2007:12(3):175-94.

40. Harvey LA. Physiotherapy rehabilitation for people with spinal cord injuries. J Physiother. 2016:62(1):4-11.

41. Ribot-Ciscar E, Butler JE, Thomas CK. Facilitation of triceps brachii muscle contraction by tendon vibration after chronic cervical spinal cord injury. J Appl Physiol. 2003;94(6):2358-67. 
42. Cotey D, Hornby TG, Gordon KE, Schmit BD. Increases in muscle activity produced by vibration of the thigh muscles during locomotion in chronic human spinal cord injury. Exp Brain Res. 2009;196(3):361-74.

43. Collins DF. Central contributions to contractions evoked by tetanic neuromuscular electrical stimulation. Exerc Sport Sci Rev. 2007;35(3):102-9.

44. Gondin J, Giannesini B, Vilmen C, Dalmasso C, le Fur Y, Cozzone PJ, et al. Effects of stimulation frequency and pulse duration on fatigue and metabolic cost during a single bout of neuromuscular electrical stimulation. Muscle Nerve. 2010;41(5):667-78.

45. Wegrzyk J, Foure A, Vilmen C, Ghattas B, Maffiuletti NA, Mattei JP, et al. Extra forces induced by wide-pulse, high-frequency electrical stimulation: occurrence, magnitude, variability and underlying mechanisms. Clin Neurophysiol. 2015;126(7):1400-12.

46. Magalhaes FH, Kohn AF. Vibration-induced extra torque during electricallyevoked contractions of the human calf muscles. J NeuroEng Rehabil. 2010;7

47. Trajano GS, Seitz LB, Nosaka K, Blazevich AJ. Can passive stretch inhibit motoneuron facilitation in the human plantar flexors? J Appl Physiol. 2014; 117(12):1486-92.

48. Bongiovanni $L G$, Hagbarth $K E$. Tonic vibration reflexes elicited during fatigue from maximal voluntary contractions in man. J Physiol-London. 1990;423:1-14.

49. Neyroud D, Armand S, De Coulon G, Da Silva SRD, Wegrzyk J, Gondin J, et al. Wide-pulse-high-frequency neuromuscular electrical stimulation in cerebral palsy. Clin Neurophysiol. 2016;127(2):1530-9.

50. Bochkezanian V, Newton RU, Trajano GS, Vieira A, Pulverenti TS, Blazevich AJ. Effect of tendon vibration during wide-pulse neuromuscular electrical stimulation (NMES) on the decline and recovery of muscle force. BMC Neurol. 2017;17(1):82.

51. Bergquist AJ, Wiest MJ, Collins DF. Motor unit recruitment when neuromuscular electrical stimulation is applied over a nerve trunk compared with a muscle belly: quadriceps femoris. J Appl Physiol. 2012;113(1):78-89.

52. Bax L, Staes F, Verhagen A. Does neuromuscular electrical stimulation strengthen the quadriceps femoris? Syst Rev Random Controlled Trials Sports Med. 2005;35(3):191-212.

53. Edgerton VR, Roy RR. Activity-dependent plasticity of spinal locomotion: implications for sensory processing. Exerc Sport Sci Rev. 2009;37(4):171-8.

54. Edgerton VR, Tillakaratne NJ, Bigbee AJ, de Leon RD, Roy RR. Plasticity of the spinal neural circuitry after injury. Annu Rev Neurosci. 2004;27: 145-67.

55. Bergquist AJ, Clair JM, Lagerquist O, Mang CS, Okuma Y, Collins DF. Neuromuscular electrical stimulation: implications of the electrically evoked sensory volley. Eur J Appl Physiol. 2011;111(10):2409-26.

56. Collins DF, Burke D, Gandevia SC. Large involuntary forces consistent with plateau-like behavior of human motoneurons. J Neurosci. 2001;21(11):4059-65.

57. Fallon JB, Macefield VG. Vibration sensitivity of human muscle spindles and Golgi tendon organs. Muscle Nerve. 2007;36(1):21-9.

58. Rehabilitation LJS. Following brain damage: some neurophysiological mechanisms. Physiological correlates of clinically observed changes in posture and tone following lesions of the central nervous system. Int Rehabil Med. 1981;4(4):195-9.

59. Xia R, Rymer WZ. Reflex reciprocal facilitation of antagonist muscles in spinal cord injury. Spinal Cord. 2005;43(1):14-21.

60. Biering-Sorensen $B$, Kristensen IB, Kjaer M, Biering-Sorensen F. Muscle after spinal cord injury. Muscle Nerve. 2009;40(4):499-519.

61. Kim HE, Corcos DM, Hornby TG. Increased spinal reflex excitability is associated with enhanced central activation during voluntary lengthening contractions in human spinal cord injury. J Neurophysiol. 2015;114(1):427-39.

62. Gordon T, Mao J. Muscle atrophy and procedures for training after spinalcord injury. Phys Ther. 1994;74(1):50-60.

63. Li Y, Li X, Harvey P, Bennett D. Effects of baclofen on spinal reflexes and persistent inward currents in motoneurons of chronic spinal rats with spasticity. J Neurophysiol. 2004;92(5):2694-703.

64. Magalhaes FH, de Toledo DR, Kohn AF. Plantar flexion force induced by amplitude-modulated tendon vibration and associated soleus V/Fwaves as an evidence of a centrally-mediated mechanism contributing to extra torque generation in humans. J NeuroEng Rehabil. 2013;10:32.

65. Adams MM, Hicks AL. Spasticity after spinal cord injury. Spinal Cord. 2005; 43(10):577-86.

66. D'Amico JM, Condliffe EG, Martins KJB, Bennett DJ, Gorassini MA. Recovery of neuronal and network excitability after spinal cord injury and implications for spasticity. Front Integr Neurosci. 2014;8:36.
67. Mizrahi J. Fatigue in functional electrical stimulation in spinal cord injury. Electromyogr Kinesiol. 1997;7(1):1-2.

68. Binder-Macleod SA, Snyder-Mackler L. Muscle fatigue: clinical implications for fatigue assessment and neuromuscular electrical stimulation. Phys Ther. 1993;73(12):902-10.

69. Kent-Braun JA. Central and peripheral contributions to muscle fatigue in humans during sustained maximal effort. Eur J Appl Physiol Occup Physiol. 1999;80(1):57-63.

70. Eklund G, Hagbarth KE, Hagglund JV, Wallin EU. The late reflex responses to muscle stretch - the resonance hypothesis versus the long-loop hypothesis. J Physiol-London. 1982;326(5):79-90.

71. Kirshblum SC, Burns SP, Biering-Sorensen F, Donovan W, Graves DE, Jha A, et al. International standards for neurological classification of spinal cord injury (revised 2011). J Spinal Cord Med. 2011;34(6):535-46.

\section{Submit your next manuscript to BioMed Central and we will help you at every step:}

- We accept pre-submission inquiries

- Our selector tool helps you to find the most relevant journal

- We provide round the clock customer support

- Convenient online submission

- Thorough peer review

- Inclusion in PubMed and all major indexing services

- Maximum visibility for your research

Submit your manuscript at www.biomedcentral.com/submit
Biomed Central 